Special issue of the 2nd International Conference on Computational and Experimental Science and Engineering (ICCESEN 2015)

\title{
Crystal Structure and EPR Investigation of Pyrazinamide Containing Copper (II) 2-Nitrobenzoate Single Crystal
}

\author{
Y. Çelik ${ }^{a, *}$, E. BozkurT ${ }^{a}$, B. Karabulut ${ }^{b}$ And N. DegE ${ }^{a}$ \\ ${ }^{a}$ Ondokuz Mayss University, Department of Physics, Samsun, Turkey \\ ${ }^{b}$ Ondokuz Mayıs University, Department of Computer Engineering, Samsun, Turkey
}

\begin{abstract}
The crystal structure and paramagnetism in the pyrazinamide containing copper(II) 2-nitrobenzoate single crystal were investigated using X-ray diffraction, Fourier-transform infrared spectroscopy, and electron paramagnetic resonance techniques. X-ray diffraction analysis shows that the complex crystallizes in monoclinic system having two molecules in the unit cell. After using curve fitting method to the electron paramagnetic resonance spectra, anisotropic $g$ and hyperfine $A$ tensors were obtained. The local symmetry, spin Hamiltonian parameters, and molecular bonding coefficients of the paramagnetic $\mathrm{Cu}^{2+}$ ion were determined. Perturbation approach was applied to the spin Hamiltonian to calculate the $g$ and $A$ values.
\end{abstract}

DOI: 10.12693/APhysPolA.130.163

PACS/topics: 61.05.cp, 75.10.Dg, 76.30.-v, 78.30.-j

\section{Introduction}

Pyrazinamide (PZA) is used as a first step drug in the treatment of tuberculosis for over 50 years [1]. It shortens the total healing time as being bactericide for some specific bacteria. PZA and its derivatives are widely used due to their antimicrobial properties [1]. It is also used to reduce the duration of chemotherapy [2]. Benzoic acid is found widely in plants and animal tissues as being the essential component of vitamin B-complex [2]. It is used to raise the salicylate level in blood and is used in miticides, contrast media in urology, and in the manufacture of pharmaceuticals [3]. Electron paramagnetic resonance (EPR) technique is widely applied to transition ions, especially to $\mathrm{Cu}(\mathrm{II})$ ions, to identify the magnetic properties, local symmetry, and spin dynamics of the complexes [4-6].

In this study, we aimed to investigate the structural and magnetic properties of this novel complex of copper(II) by using X-ray diffraction (XRD), Fouriertransform infrared (FT-IR) spectroscopy and EPR methods. We have also tried to calculate theoretical values for electronic transitions and molecular bonding coefficients.

\section{Materials and methods}

All chemical reagents were analytical grade commercial products. Solvents were purified by conventional methods. KBr disks and a Vertex 80v Bruker FT-IR spectrophotometer were used to record the IR spectra in $400-4000 \mathrm{~cm}^{-1}$ region. Varian E-109C model X-band spectrometer was used for EPR measurements. Suitable single crystal was mounted on a goniometer and the spectra were recorded in three mutually perpendicular planes at $10^{\circ}$ intervals at ambient temperature. The $g$ values

\footnotetext{
${ }^{*}$ corresponding author; e-mail: yunus.celik@omu.edu.tr
}

were obtained by comparison with a diphenylpicrylhydrazyl (dpph) sample of $g=2.0036$. Reported values involve errors within \pm 0.005 for $g$ values and $\pm 0.05 \mathrm{mT}$ for hyperfine coupling constants $A$.

\subsection{Syntheses of pyrazinamide containing copper(II) 2-nitrobenzoate complex}

Aqueous solution of 2-nitrobenzoic acid $(0.67 \mathrm{~g}$, $4 \mathrm{mmol}$ ) was added into aqueous solution of $\mathrm{Cu}(\mathrm{II})$ acetate $(0.36 \mathrm{~g}, 2.0 \mathrm{mmol})$. After stirring for about $1 \mathrm{~h}$, precipitates were filtered to yield the compound $[\mathrm{Cu}(2-$ $\left.\mathrm{NB})_{2}\right] \cdot 2 \mathrm{H}_{2} \mathrm{O}$. Aqueous solution of pyrazinamide $(0.49 \mathrm{~g}$, $4 \mathrm{mmol}$ ) was added into the aqueous solution of this compound under stirring and the mixture was allowed to stand at room temperature. After about a week, blue well-shaped single crystals of the compound were obtained.

\section{Results and discussion}

\subsection{XRD analysis}

The molecular structure of the complex having the formula $\left[\mathrm{Cu}(2-\mathrm{NB})_{2}(\mathrm{PZA})_{2}\left(\mathrm{H}_{2} \mathrm{O}\right)_{2}\right]$ (NB: nitrobenzoic acid, PZA: pyrazinamide) is shown in Fig. 1. Bond lengths and

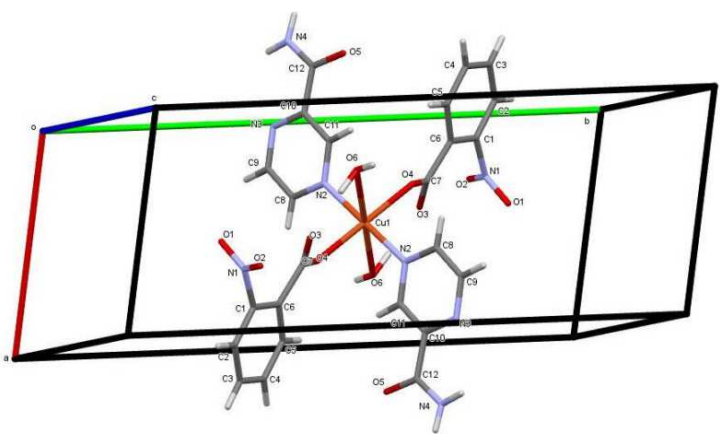

Fig. 1. Molecular structure of $\left[\mathrm{Cu}(2-\mathrm{NB})_{2}(\mathrm{PZA})_{2}-\right.$ $\left(\mathrm{H}_{2} \mathrm{O}\right)_{2}$ ] complex. Atoms are numbered with the exception of hydrogens. 
inter-bond angles obtained from the the crystallographic data are given in Table I. The $\mathrm{Cu}(\mathrm{II})$ ion is coordinated to $\mathrm{O} 4$ atoms of two 2-nitrobenzoic acids and N2 atoms of two pyrazinamide ligands forming the equatorial plane. Axially it is coordinated to two aqua ligands and completes the coordination sphere as distorted octahedron. The bond lengths of $\mathrm{Cu} 1-\mathrm{O} 6$ in axial positions are longer than those of $\mathrm{Cu} 1-\mathrm{O} 4$ and $\mathrm{Cu} 1-\mathrm{N} 2$ in equatorial plane and hence the symmetry of the complex is D4h. In most of the $\mathrm{Cu}$ (II) complexes, axial elongation is observed and can be explained in view of Jahn and Teller [7].

TABLE I

Crystal data, selected bond lengths and angles of $[\mathrm{Cu}(2-$ $\mathrm{NB})_{2}(\mathrm{PZA})_{2}\left(\mathrm{H}_{2} \mathrm{O}\right)_{2}$ ] complex.

\begin{tabular}{c|c}
\hline \hline Chemical formula & $\mathrm{C}_{24} \mathrm{H}_{22} \mathrm{CuN}_{8} \mathrm{O}_{12}$ \\
Molecular weight & 678.03 \\
Crystal system, space group & Monoclinic, P21/c \\
Temperature $[\mathrm{K}]$ & 293 \\
$a, b, c[\AA]$ & $119.549(3)$ \\
$\beta\left[^{\circ}\right]$ & $1390.77(11)$ \\
$V\left[^{3}\right]$ & 2 \\
$Z$ & Bond angles $\left[^{\circ}\right]$ \\
\hline Bond lengths $[\AA]$ & $\mathrm{O} 4-\mathrm{Cu} 1-\mathrm{N} 2: 91.02(6)$ \\
$\mathrm{Cu} 1-\mathrm{O} 4:$ O $1.9687(12)$ & $\mathrm{N} 2-\mathrm{Cu} 1-\mathrm{N} 2: 180$ \\
$\mathrm{Cu} 1-\mathrm{N} 2: 2.0133(15)$ & $\mathrm{O} 4-\mathrm{Cu} 1-\mathrm{O} 4: 180$
\end{tabular}

\subsection{FT-IR results}

FT-IR spectrum of $\left[\mathrm{Cu}(2-\mathrm{NB})_{2}(\mathrm{PZA})_{2}\left(\mathrm{H}_{2} \mathrm{O}\right)_{2}\right]$ single crystal was recorded at room temperature and is shown in Fig. 2. Some of the main bands in the spectrum were

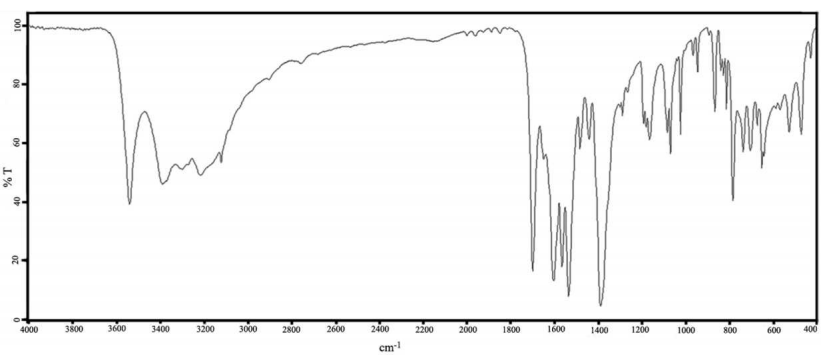

Fig. 2. FT-IR spectrum of $\left[\mathrm{Cu}(2-\mathrm{NB})_{2}(\mathrm{PZA})_{2}\left(\mathrm{H}_{2} \mathrm{O}\right)_{2}\right]$ complex at room temperature.

explained and compared with the results of similar works. The strong absorption bands at 1601 and $1563 \mathrm{~cm}^{-1}$ correspond to the carbonyl asymmetric stretching bands of 2-nitrobenzoic acid ligand. Shah et al. observed these bands at 1590 and $1550 \mathrm{~cm}^{-1}$ [8]. Similarly, the symmetric stretching mode of the carbonyl group was observed at $1387 \mathrm{~cm}^{-1}$ while Shah et al. found them at 1388 and $1362 \mathrm{~cm}^{-1}$ in their study [8]. The strong band appearing at $1533 \mathrm{~cm}^{-1}$ in the complex is associated with asymmetric stretching vibrations of $\mathrm{NO}_{2}$ group. This mode was observed at $1568 \mathrm{~cm}^{-1}$ in [9]. A very strong absorption band at $1697 \mathrm{~cm}^{-1}$ was assigned to mode $\nu(\mathrm{C}=\mathrm{O})$ related to pyrazinamide ligand. It was observed at around $1700 \mathrm{~cm}^{-1}$ in the literature $[10,11]$. The strong absorption band at $3539 \mathrm{~cm}^{-1}$ corresponds to the asymmetric vibrations of $\mathrm{NH}_{2}$ in pyrazinamide ligand. This band was observed at $3500 \mathrm{~cm}^{-1}$ and $3414 \mathrm{~cm}^{-1}$ in some pyrazinamide complexes [10, 11]. A medium absorption band at $3389 \mathrm{~cm}^{-1}$ was assigned to the symmetric deformation mode of $\mathrm{NH}_{2}$ and in literature it was observed at 3368, 3386 and $3376 \mathrm{~cm}^{-1}[10,11]$.

\subsection{EPR study}

EPR spectra of both single crystal and powdered sample of the title complex were recorded at room temperature and are shown in Fig. 3. $\mathrm{Cu}(\mathrm{II})$ ion has $\mathrm{d}^{9}$ configuration having one unpaired electron. Moreover, it has a nuclear spin $I=3 / 2$ and so has a dipolar interaction with the unpaired electron. This interaction is also known as hyperfine interaction. When such an interaction is present in paramagnetic systems, one can expect four lines in an EPR spectrum since the magnetic spin quantum number $\left(m_{I}=+I I-1, \ldots,-I+1,-I\right)$ of the nucleus has four different values $(+3 / 2,+1 / 2,-1 / 2,-3 / 2)$. If the system is anisotropic, i.e. its properties depend on the orientation of the applied (external) magnetic field, then the positions of the lines and the distance between two adjacent lines (hyperfine splitting constant) change for each angle in the recorded EPR spectrum $[12,13]$. Hence, at some orientations, the lines overlap and cause the number of lines to decrease (Fig. 3a).

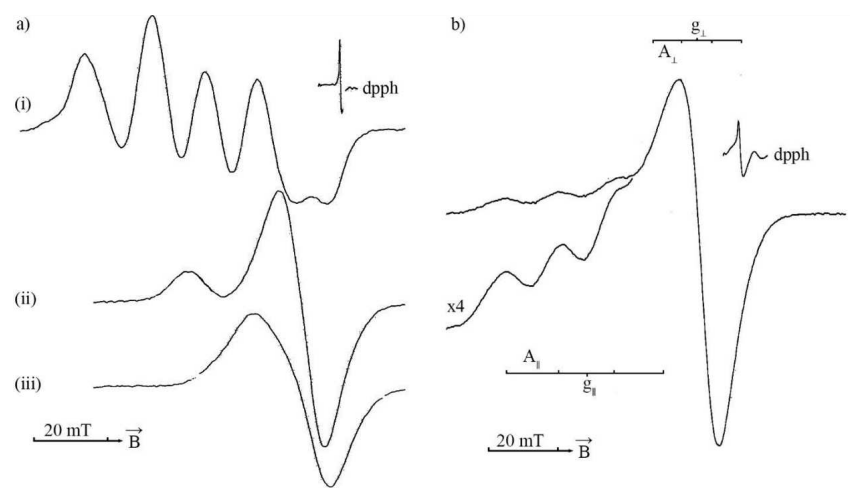

Fig. 3. (a) EPR spectra of $\left[\mathrm{Cu}(2-\mathrm{NB})_{2}(\mathrm{PZA})_{2}\left(\mathrm{H}_{2} \mathrm{O}\right)_{2}\right]$ single crystal at three different orientations in $a^{*} b$ plane: (i) $20^{\circ}$, (ii) $70^{\circ}$, (iii) $90^{\circ}$ relative to $a^{*}$ axis. (b) EPR spectrum of powdered $\left[\mathrm{Cu}(2-\mathrm{NB})_{2}(\mathrm{PZA})_{2}\left(\mathrm{H}_{2} \mathrm{O}\right)_{2}\right]$ complex.

On the other hand, unpaired electrons in the complex interact with the external magnetic field and have an energy difference between spin states (electron Zeeman splitting). When the energy of microwave radiation sent to the sample is equal to the energy gap between spin states, resonance condition is reached and transitions from lower spin state to the higher state take place until the steady state is reached. The spins at excited 


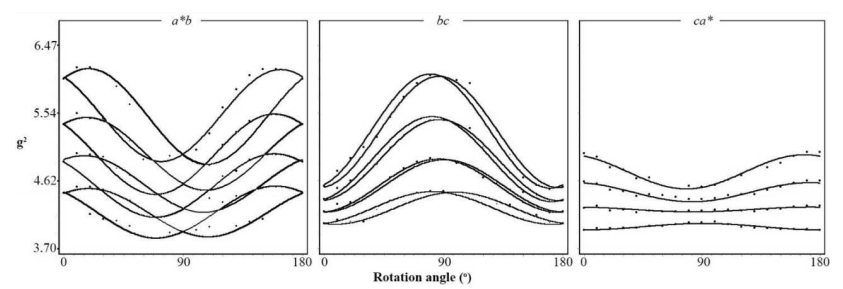

Fig. 4. Plot of $g^{2}$ vs rotation angle for the complex under study.

state return to the ground state by giving their energy to either crystal lattice or other spins. The duration of de-excitation is called the relaxation time and the shorter this time is, the wider is the EPR line-width. Furthermore, if the unpaired electron density in the complex is large, then the line-widths in the EPR spectra are wide [12]. Thus, the line-widths in the EPR spectra of the title complex can be explained in these terms.

The spin Hamiltonian including only electron Zeeman and hyperfine interactions can be described as:

$$
\mathcal{H}=\beta \boldsymbol{H} \widehat{g} \boldsymbol{S}+\boldsymbol{S} \widehat{A} \boldsymbol{I} .
$$

To construct the $\widehat{g}$ and $\widehat{A}$ tensors for the $[\mathrm{Cu}(2$ $\mathrm{NB})_{2}(\mathrm{PZA})_{2}\left(\mathrm{H}_{2} \mathrm{O}\right)_{2}$ ] complex, $g^{2}$ values of all recorded EPR spectra in three axes were plotted against rotation angle, as in Fig. 4. Least squares technique was used for curve fitting to obtain principal values (eigenvalues) and direction cosines (eigenvectors) of the tensors [13]. The results show that the local symmetry of the $\mathrm{Cu}(\mathrm{II})$ ion is rhombic and the $\mathrm{Cu}$ (II) ion has two sites in the complex. All these values are listed in Table II. For the powdered complex the $g$ and $A$ values were obtained from Fig. 3b as follows: $g_{z}=2.299, g_{x}=g_{y}=2.080$, $A_{z}=15.47 \mathrm{mT}, A_{x}=A_{y}=8.43 \mathrm{mT}$.

TABLE II

Direction cosines and principal values of $g^{2}$ and $A^{2}$ tensors in $[\mathrm{Cu}(2$ $\mathrm{NB})_{2}(\mathrm{PZA})_{2}\left(\mathrm{H}_{2} \mathrm{O}\right)_{2}$ ] single crystal.

\begin{tabular}{|c|c|c|c|c|c|c|c|c|}
\hline \multirow{3}{*}{ Site } & \multicolumn{4}{|c|}{$g^{2}$ tensor } & \multicolumn{4}{|c|}{$\overline{A^{2} \text { tensor }}$} \\
\hline & \multirow{2}{*}{$\begin{array}{c}\text { Principal } \\
\text { values }\end{array}$} & \multicolumn{3}{|c|}{ Direction cosines } & \multirow{2}{*}{$\begin{array}{c}\text { Principal } \\
\text { values }[\mathrm{mT}]\end{array}$} & \multicolumn{3}{|c|}{ Direction cosines } \\
\hline & & $a^{*}$ & $\mathrm{~b}$ & $\mathrm{c}$ & & $\mathrm{a}^{*}$ & $\mathrm{~b}$ & $\mathrm{c}$ \\
\hline \multirow{3}{*}{ I } & 2.286 & 0.9603 & 0.2776 & 0.0249 & 15.93 & 0.9103 & 0.3761 & 0.1727 \\
\hline & 2.087 & -0.2551 & 0.9115 & -0.3224 & 11.63 & -0.3172 & 0.9020 & -0.2925 \\
\hline & 2.064 & -0.1122 & 0.3033 & 0.9462 & 3.53 & -0.2658 & 0.2115 & 0.9405 \\
\hline \multirow{3}{*}{ II } & 2.299 & 0.9298 & -0.3522 & 0.1062 & 16.79 & 0.9102 & -0.2378 & 0.3389 \\
\hline & 2.073 & 0.2674 & 0.8454 & 0.4623 & 11.87 & 0.2375 & 0.9704 & 0.0432 \\
\hline & 2.062 & -0.2526 & -0.4014 & 0.8803 & 1.70 & -0.3392 & 0.0410 & 0.9398 \\
\hline
\end{tabular}

TABLE III

Theoretical and experimental comparison of molecular bonding coefficients, spin Hamiltonian parameters, and d-d transition energies for $\left[\mathrm{Cu}(2-\mathrm{NB})_{2}(\mathrm{PZA})_{2}\left(\mathrm{H}_{2} \mathrm{O}\right)_{2}\right]$ single crystal.

\begin{tabular}{c|c|c|c|c|c|c|c|c|c|c}
\hline \hline$\alpha_{0}^{2}$ & $\beta_{1}^{2}$ & $\beta_{0}^{2}$ & $\kappa$ & $g_{\perp}$ & $g_{||}$ & $\left|A_{\perp}\right|[\mathrm{mT}]$ & $\left.\left|A_{\| \mid}\right| \mathrm{mT}\right]$ & $B_{1 g} \rightarrow B_{2 g}\left[\mathrm{~cm}^{-1}\right]$ & $B_{1 g} \rightarrow E_{g}\left[\mathrm{~cm}^{-1}\right]$ & Reference \\
\hline 0.66 & $\approx 1$ & $\approx 1$ & 0.53 & 2.080 & 2.299 & 8.43 & 15.47 & 15120 & 18195 & $a$ \\
0.67 & $\approx 1$ & $\approx 1$ & 0.56 & 2.049 & 2.300 & 7.12 & 14.56 & 15120 & 18850 & $b$ \\
\hline
\end{tabular}

$a$ : Experimental, $b$ : Theoretical

In order to calculate the molecular bonding coefficients, one needs to apply the perturbation approach to spin Hamiltonian and carry out further calculations for energies of $d-d$ transitions in the complex. The required formulas and values were taken from Refs. [14-18] and the coefficients of the molecular bonding were calculated. To fit the theoretical results to the experimental values the same approach was used as in [14-18]. Both the theoretical and experimental results are listed in Table III. From the results one can conclude that in plane $\sigma$ bonding $\left(\alpha_{0}^{2}\right)$ is dominantly covalent, while in plane $\left(\beta_{1}^{2}\right)$ and out of plane $\left(\beta_{0}^{2}\right) \pi$ bonds are almost ionic in nature.

\section{Conclusions}

Molecular structure and magnetic properties of $[\mathrm{Cu}(2-$ $\left.\mathrm{NB})_{2}(\mathrm{PZA})_{2}\left(\mathrm{H}_{2} \mathrm{O}\right)_{2}\right]$ single crystal were studied using XRD, FT-IR and EPR techniques. The crystal belongs to monoclinic system and magnetically the local symmetry of the paramagnetic $\mathrm{Cu}(\mathrm{II})$ ion is found to be rhombic. The spin Hamiltonian parameters with the molecular bonding coefficients were also calculated. Theoretical formulas are used to verify the experimental results. 


\section{Supplementary data}

Crystallographic data for the structure in this paper have been deposited at the Cambridge Crystallographic Data Centre as the supplementary publication № CCDC 1434905. Copies of the data can be obtained free of charge, on application to CCDC, 12 Union Road, Cambridge, CB12 1EZ, UK, fax: +44 1223366 033, or e-mail: deposit@ccdc.cam.ac.uk

\section{Acknowledgments}

This work was supported by Ondokuz Mayıs University as a scientific research project grant № PYO.FEN.1901.13.004

\section{References}

[1] T. Jurca, E. Marian, Farmacia 2, 247 (2009).

[2] C. Miyagi, N. Yamane, B. Yogesh, H. Ano, T. Takashima, Diagn. Micr. Infec. Dis. 48, 111 (2004).

[3] M. Ramalingam, N. Sundaraganesan, H. Saleem, J. Swaminathan, Spectrochim. Acta A 71, 23 (2008).

[4] Y. Çelik, E. Bozkurt, İ. Uçar, B. Karabulut, Chem. Phys. Lett. 514, 278 (2011).

[5] S. Idziak, S.K. Hoffmann, J. Goslar, Acta Phys. Pol. A 108, 177 (2005).
[6] Y. Yerli, Spectrochim. Acta A 66, 1288 (2007).

[7] H.A. Jahn, E. Teller, Proc. R. Soc. Lond. A 161, 220 (1937).

[8] F.A. Shah, S. Ali, S. Shahzadi, C. Rizzoli, S. Ianelli, J. Chem. Crystallogr. 41, 1249 (2011).

[9] R. Mrozek, T. Glowiak, J. Chem. Crystallogr. 34, 153 (2004).

[10] M.A.S. Goher, F.A. Mautner, Polyhedron 19, 601 (2000).

[11] S. Akyuz, L. Andreeva, B. Minceva-Sukarova, G. Basar, J. Molecular Struct. 834-836, 399 (2007).

[12] A. Abragam, B. Bleaney, Electron Paramagnetic Resonance of Transition Ions, Oxford University Press, Oxford 1970.

[13] B. Karabulut, R. Tapramaz, Radiat. Phys. Chem. 55, 331 (1999).

[14] P. Budhani, S.A. Iqbal, S.M.M. Bhattacharya, L. Mitu, J. Saudi Chem. Soc. 14, 281 (2010).

[15] D. Attanasio, J. Magn. Reson. 26, 81 (1977).

[16] W.-L. Feng, K. Wang, F. Zhao, J.-Y. Xue, J. Phys. Chem. Solid. 75, 787 (2014).

[17] D. Kivelson, R. Neiman, J. Chem. Phys. 35, 149 (1961).

[18] D.J. Newman, B. Ng, Rep. Prog. Phys. 52, 699 (1989). 